\title{
Spinal cord neural stem cells heterogeneity in postnatal development
}

\author{
Jelena BAN ${ }^{*}$ and Miranda MLADINIC \\ Laboratory for Molecular Neurobiology, Department of Biotechnology, University of Rijeka, Radmile Matejčić 2, 51000 Rijeka, Croatia \\ ${ }^{*}$ Correspondence: jelena.ban@biotech.uniri.hr \\ https://doi.org/10.37175/stemedicine.v1i1.19
}

\begin{abstract}
Neural stem cells are capable of generating new neurons during development as well as in the adulthood and represent one of the most promising tools to replace lost or damaged neurons after injury or neurodegenerative disease. Unlike the brain, neurogenesis in the adult spinal cord is poorly explored and the comprehensive characterization of the cells that constitute stem cell neurogenic niche is still missing. Moreover, the terminology used to specify developmental and/or anatomical central nervous system regions, where neurogenesis in the spinal cord occurs, is not consensual and the analogy with the brain is often unclear. In this review, we will try to describe the heterogeneity of the stem cell types in the spinal cord ependymal zone, based on their origin and stem cell potential. We will also consider specific animal in vitro models that could be useful to identify the "right" stem cell candidate for cell replacement therapies.
\end{abstract}

Keywords: Neural stem cells · Radial glia $\cdot$ Ependymal cells $\cdot$ Development $\cdot$ Spinal cord injury

\section{Introduction}

Spinal cord injury (SCI) of traumatic or non-traumatic origin is a devastating neurological condition, with severe motor disfunction and other deficits. Recent advances in SCI research have enabled development of different therapeutic strategies trying to counteract the detrimental consequences of the processes involved in secondary injury, or trying to replace damaged or lost neurons at the place of injury, using cell transplantation therapies as reviewed recently in (1-3). However, the clinical translation of the therapeutic strategies with substantial benefits for patients has thus far been elusive, setting the necessity for better understanding of both molecular and cellular mechanisms involved in the pathophysiology of the SCI and stem cell biology.

Neural stem cells (NSC) are promising source of donor cells that could be used in cell transplantation strategies to overcome the incapacity of the central nervous system (CNS) to repair and regenerate after injury. Because of their limited accessibility and ethical concerns regarding the use of human embryonic stem cells (ESC), induced pluripotent stem cells (iPSCs), from which patient-specific

(c) The Author(s). 2019 This is an Open Access article distributed under the terms of the Creative Commons License (http://creativecommons.org/licenses/by/4.0/) which permits unrestricted use, distribution, and reproduction in any medium or format, provided the original work is properly cited.
NSC could be produced, offer the exceptional opportunity for development of personalized cell replacement therapies (4-7). Generation of the human iPSCs was first reported in 2007, only one year after the remarkable discovery that murine fibroblasts can be reprogrammed into pluripotent cell state $(8,9)$. Since then, research in the field had enormous growth and iPSCs have also been widely considered for the treatment of the SCI $(3,10)$, with the first-in-human clinical study to launch for subacute SCI (11).

However, several concerns have arisen regarding their clinical use, related to the reprogramming method (alternatives to viral vectors are necessary) and the low efficiency for both reprogramming rate and differentiation yield. Probably, the most important issue is the fact that the reprograming procedure, with high number of passages in vitro required to reach pluripotent cell state, carries high risk of generation of new mutations. This can lead to tumorigenesis in addition to retainment of patientspecific mutations $(6,12)$. Chemical reprogramming with small molecules, mostly epigenetic modulators, succeeded to produce iPSCs in mice (13), as well as chemically induced NSC (14) and neurons $(15,16)$. Transdifferentiation, i.e. direct conversion of somatic cells into another differentiated CNS cell lineage occurs without induction of pluripotency by gene delivery and is therefore considered safer for clinical application than 
human iPSCs.

Valid alternative to the cell transplantation strategies is the use of endogenous spinal cord stem cells to replace the lost or damaged neurons in loco (without need of transplantation). The endogenous spinal cord stem cells are tissue-specific stem cells, already committed to neural phenotype. Therefore, there would be no need for them to reach the pluripotent state. The ideal solution would be to instruct those cells to produce new neurons that will integrate into existing networks, by controlling their differentiation mechanisms.

The premise for the use of endogenous stem cells is their complete characterization and deep knowledge of the reprogramming mechanisms. The characterization of spinal cord stem cell niche(s) is still incomplete in terms of cell types, for most of which the exclusive markers are missing, and in terms of their differentiation potential and their localization. Moreover, to favor neuroregeneration after SCI or neurodegenerative diseases, it is necessary to unblock the inhibitory components in the extracellular space and possibly, to also "reprogram" glial cells to favor the regeneration-permissive anti-inflammatory phenotype (17-20).

Here we will describe what is known about the cellular composition of the spinal region around the central canal, which is considered stem cell niche that persists in adulthood. This region is composed of heterogeneous cell populations, some of which are still not fully identified (21). This heterogeneity arises very early in the development and therefore it is crucial to track and unveil the developmental origin of each of those different cell subtypes, in order to exploit them for therapeutic purposes.

\section{Overlapping stem cell terminology}

There are many terms used to describe a variety of cells that appear during highly dynamic developmental processes of cell proliferation and differentiation. Specific terms are assigned to particular developmental stage of a particular CNS region and in a particular animal species. Neurogenesis during development and adulthood is best characterized in the cortex. Although this knowledge could be applicable to other CNS regions (22), including spinal cord, the differences and the analogy are not completely clear and there are still ongoing debates.

NSC is the generic and most frequent term to describe multipotent stem cells that can generate all four CNS lineages with ectodermal origin: neurons, astrocytes, oligodendrocytes and ependymal cells (22-24). The term "neural" denotes the loss of pluripotency, characteristic of ESC or iPSCs only. NSC are found in both developing and adult nervous system. By definition, NSC are precursors of neurons and glia and therefore should be identical to neural progenitor (or precursor) cells (NPCs) (22). However, some researchers distinguish NPCs as cells with limited self-renewing capacity, contrary to NSC, considered to have prolonged self-renewal and broader differentiation potential $(21,25)$. The combined term stem/ progenitor cell (SPC) has also been used (26-28).
Moreover, very often there is a "transitory" stem cell state: for example, NSC or NPC can give rise to distinct subpopulation of neuron and glial precursor cells, respectively. These transit amplifying cells (22) or intermediate progenitor cells (IPC) are considered to be multipotent stem cells, able to generate neurons (nIPC), astrocytes (aIPC) and oligodendrocytes (oIPC) (22) or bipotent (29) as well as unipotent stem cells, expressing transcription factor Tbr2, capable of generating neurons only (30-32). IPC in developing cortex are also called basal progenitors (25) indicating their anatomical position. In addition to these two currently interchangeable terms, several other adjectives such as subependymal or extraventricular, abventricular, non-surface, subventricular, etc. (33) were used to describe these mitotic NPCs.

The term "basal progenitor" unavoidably leads to radial glia (RG), "universal" precursors of all CNS cell types of the neuroectodermal lineage. $\mathrm{RG}$ are therefore both NSC and NPC (22,33). They develop from neuroepithelial cells (NEC) or neuroepithelial progenitors (25) early in embryonic development, at the onset of neurogenesis (E9/E10 in mouse) $(22,25)$ and generate mitotic NPC or IPC by asymmetric division. RG morphology is typically bipolar: one long process that projects radially and reaches pial (outer) surface while the shorter one faces the lumen of the ventricle; cell body is confined to the apical region that will become the ventricular zone (VZ) (25). For a long time they were seen solely as scaffolds, i.e. structural support for neuronal migration, while now their neuroand gliogenic potential is widely accepted (34-36).

Some researchers consider them to be present only during embryonic development and absent in the mature brain (37), while others identify them as adult NSC $(23,35,38)$ or similarly, consider that the adult NSC are "RG-like" $(33,38)$. Although RG are historically described as fetal glia (33), indicating their glial nature, and because of the presence of the glial fibrillary acidic protein (GFAP), astrocyte-specific glutamate transporter (GLAST), glutamine synthetase, S100 $\beta$ or vimentin expression $(21,34,39)$, they are sometimes classified as astrocytes' subtype (37). RG are heterogeneous cell population consisting of bipolar apical progenitors, confined to the $\mathrm{VZ}$, and basal RG that migrate away from VZ, also named translocating, transitional, transforming or intermediate RG (33). Late neurogenesis is followed by gliogenesis around E17.5 in mouse (25) which continues postnatally. During gliogenesis, bipolar RG progressively become uniand multipolar, assuming astrocytes' morphology (22).

In summary, the present NSC terminology reflects the intrinsic complexity and heterogeneity of developing CNS cells. There is however a need for consensus and clear, unified terminology (33).

Recently, single cell biology, with single cell transcriptomics in particular, is emerging as a revolutionary field that questions our traditional views on distinguishing between cell type and cell state (29). In the proposed model, called 'periodic table of cell types', where cell types are defined according to their transcription factors' expression profile, the sequence of events, i.e. cells that appear during CNS 

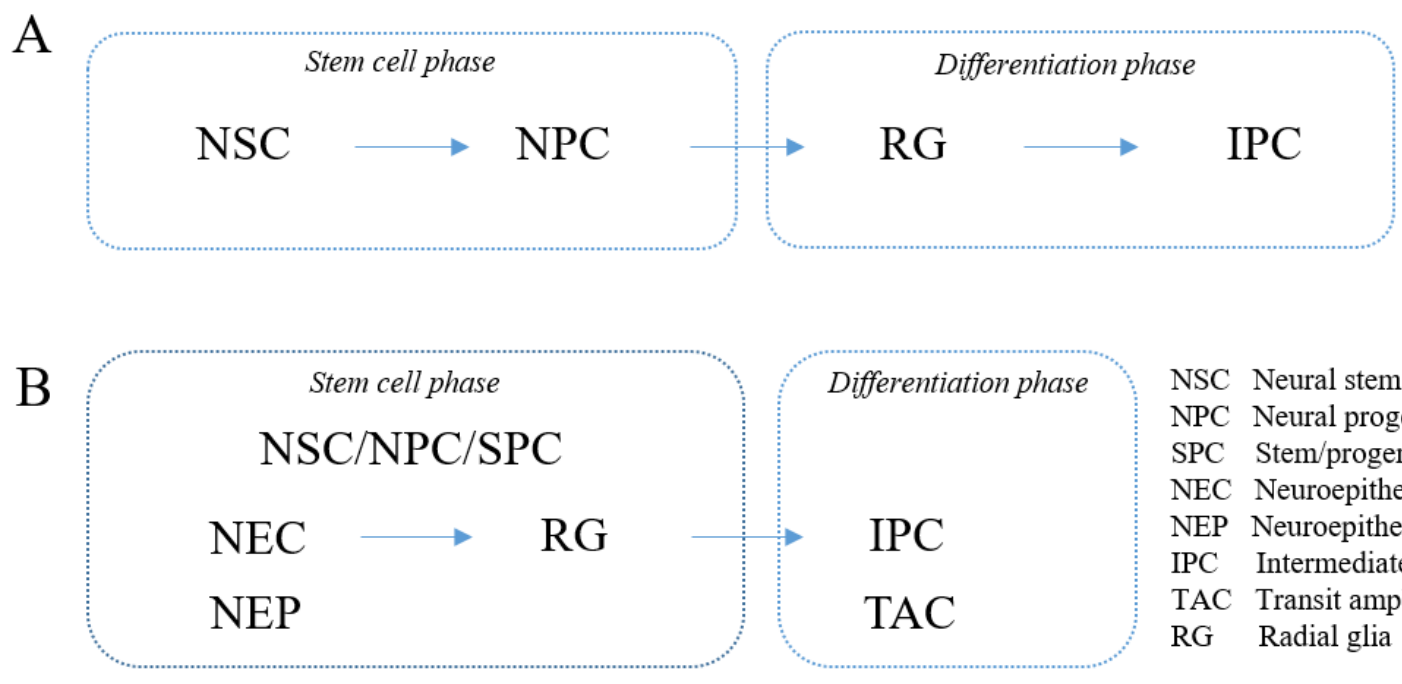

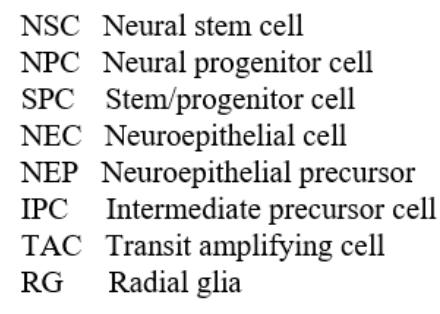

NSC Neural stem cell

NPC Neural progenitor cell

SPC Stem/progenitor cell

NEC Neuroepithelial cell

NEP Neuroepithelial precursor

IPC Intermediate precursor cell

RG Radial glia

Figure 1. Terminology used to describe cells that appear during CNS development. (A) Recently proposed method in which a periodic table of cell types is proposed with stem cell and differentiation phase (29). (B) Additional terms collected from the literature. NSC, Neural stem cell; NPC, Neural progenitor cell; SPC, stem/progenitor cell; NEC, Neuroepithelial cell; NEP, Neuroepithelial precursor; RG, Radial glia; IPC, Intermediate precursor cell; TAC, Transit amplifying cell.

development, is: NSC, NPC, RG, IPC, ending with differentiated cells (neurons, astrocytes, oligodendrocytes and EC) (29), as summarized in Figure 1A. NSC and NPC both belong to "stem cell phase" while RG and IPC are considered to be in "differentiation phase", according to this model. In Figure 1B, the most frequently used (and overlapping) terms are shown and a more inclusive version is proposed.

Most of our knowledge about developing and adult NSC niches comes from studies in the cerebral cortex, and in the next section we will try to apply this knowledge to the spinal cord.

\section{Spinal cord central canal stem cell niche}

In the spinal cord, unlike the cortex, NSC are less well understood. The pool of stem cells is considered to be mainly confined to the region surrounding the central canal, with EC representing a main cell type. Stem cells are also found dispersed in the spinal cord tissue outside the ependymal region, with more restricted proliferative potential (21).

The ependymal region is composed of different cells facing the lumen as well as cells in subependymal position, reminiscent of pseudostratified epithelium. The important difference, when compared to the brain, is the absence in the spinal cord of the distinct subependymal layer such as subventricular zone (SVZ) $(21,26)$. Moreover, in vivo neurogenesis in the adult spinal cord has not been reported yet. For a long time, the region surrounding the spinal central canal was thought to be composed of a single layer of densely packed ciliated and cuboidal, epithelial-like secretory cells $(21,23,40)$, while today it becomes clear that these cells are not homogeneous at all: in addition to $\mathrm{EC}$, still uncharacterized cells with unknown function exist (21).

EC, together with astrocytes, oligodendrocytes and microglia, are one of the four main types of (neuro) glia (41), although sometimes are included into astroglia (37). $\mathrm{EC}$ are cells of neuroectodermal origin, deriving from proliferating NEC around the central lumen of the caudal neural tube, from which the central canal and spinal cord develops. At the onset of neurogenesis, NEC will give rise to $R G(22,40)$, and at that stage NEC are already heterogeneous cell population in terms of stemness and differentiation potential (21). In the brain, $\mathrm{RG}$ give rise to EC between E14-E16 in mouse, while in the spinal cord they seem to appear later, earliest at E15.5 (and around E18 in rats) $(40,42,43)$. Even though EC are generated during embryonic age, their differentiation and maturation, characterized for example by the formation of cilia, occur only after birth, in the first postnatal week (32). Proliferation of ependymocytes continues for several weeks after birth, necessary for the extension of the growing spinal cord. At 9 weeks after birth in mouse and 12-13 weeks in rats, the spinal cord stops elongating, cell proliferation stops and EC enters a quiescent state $(21,28,40)$. Adult ependymocytes in the spinal cord face the lumen of central canal and, as those of the brain ventricles, produce cerebrospinal fluid $(\mathrm{CSF})$ and regulate its composition and movement.

The adult ependymal region is composed of lateral and polar domains. In addition to the cuboidal ependymocytes, distributed laterally, another cell type is present: tanycytes, also referred to as radial ependymocytes or radial ependymal cells (21). Their cell body is situated in ependymal or subependymal region, the apical process is in contact with CSF and the long basal process with blood vessels. Their main functions are uptake and transport of substances (regulation of CSF composition) as well as regulation of vasodilatation of spinal cord vessels, as indicated by the expression of vasoactive intestinal 
polypeptide (VIP) (21).

Tanycytes are also found in polar domains of the central canal where high number of cells present radial morphology and express GFAP. This third cell type is known as radial dorsal cells or dorsal tanycytes or ependymoglial cells $(21,44)$. In common with all EC of the central canal, they express nestin and VIP, while with ependymocytes share SOX2 and vimentin expression. Subpopulation of these cells show additional expression of NSC markers such as BLBP and CD15 (21). Moreover, expression of transcription factor ZEB1, a mesenchymal cell marker, was reported (28).

GFAP + cells, with shorter basal process and in smaller number, are also found in the ventral part of the central canal and occasionally in lateral part, with cell bodies placed in subependymal region (21). In addition to different EC, there are also sporadically distributed neurons that send projection in the central canal having their soma situated in ependymal or subependymal region, called CSF contacting neurons and finally, some still unidentified cells are observed $(21,40)$.

As can be noted, ependymal region of the spinal cord is composed of a variety of cells with distinct morphologies and with significant overlap in expression profiles between different cell types, which represent a challenge in proper classification. For instance, tanycytes are considered to be a subtype of ependymal cells in the mouse spinal cord $(21,44)$, but evidences showing differences in the expression profile of the better-characterized brain counterparts have emerged $(40,44,45)$. Moreover, their similarity with RG (both share radial morphology and express common markers that persist in the adult spinal cord) makes the distinction between different ependymal cell types less clear.

\section{Are EC/ependymocytes stem cells?}

The presence of NSC markers such as SOX2, CD15, CD133, nestin, vimentin, BLBP, GFAP and others $(21,40,45)$ in the adult ependymal region opens another controversial issue: are EC adult NSC of the spinal cord? Do RG (or RG-like cells) persist in the adult spinal cord? Why adult neurogenesis in the spinal cord is more limited than brain neurogenesis?

The central canal is considered spinal cord stem cell niche but the degree of "stemness" of ependymocytes is still discussed. In the brain, the most accepted view is that the only in vivo proliferating NSC/NPC capable of neurogenesis are B1 cells, RG-like subpopulation of astrocytes in the $\operatorname{SVZ}(25,32,33,43,46)$. Therefore, EC of the brain are not considered to be NSC but rather unipotent cells (46).

After injury, ependymal cells become activated, proliferate, migrate and differentiate into astrocytes and oligodendrocytes, contributing to scar formation $(21,26-28,44,47)$.

In the spinal cord, both ependymal (47) and GFAPpositive RG-like dorsal cells $(21,48)$ were investigated as putative stem cells despite the reports in which adult rodent spinal cord EC were defined as postmitotic cells (42).
In addition to the expression of NSC markers observed in vivo, in vitro experiments indicated different differentiation as well as proliferative potential of EC. In vitro stemness is evaluated with a "neurosphere assay": NSC of particular brain or spinal cord region are dissected, dissociated and cultured usually in suspension, in the presence of basic fibroblast growth factor and epidermal growth factor. After several days in culture, free-floating (neuro)spheres are formed and can be further dissociated and replated in either proliferative condition (generating secondary neurospheres) or induced to differentiate, offering the way to asses their in vitro differentiation potential. This assay was first developed in early 1990s (49) and also became one of the most frequently used. Despite its notable utility, it should be kept in mind that the in vitro differentiation potential could differ from the in vivo one, since the extracellular environment strongly influences cell behavior. Moreover, not all NSC are efficiently amplified in vitro (quiescent NSC or the specific NSC/ NPC subpopulations present in very low number could not generate neurospheres) and not all neurospheres are derived from NSC [for a review see (50)].

This assay has also provided one of the first evidences that cells in adult spinal cord generate multipotent neurospheres $(47,51)$ with both neurogenic and gliogenic potential, and this NSC property was explicitly assigned to EC (47). However, at that time, the ependymal layer was thought to be composed of homogeneous cell population, and in the same year (1999) another study showed that neurosphere-forming cells in SVZ were not EC, but astrocytes (also known as B1 cells), and that EC did not divide in vivo (52). Moreover, brain ciliated EC were defined as unipotent cells capable of forming neurospheres, but with gliogenic instead of neurogenic potential (46).

The critical role of extracellular environment was further demonstrated when spinal cord-derived dissociated neurospheres transplanted in the hippocampus generated neurons, while those transplanted in the spinal cord became only glial cells (53).

The explanation of the contrasting results obtained in the last two decades is still missing and further investigations are necessary to understand the stem cell potential of the ependymal region of the spinal cord.

\section{Opossum model to study ependymal spinal cord SPC}

In contrast to invertebrates and lower vertebrates, mammals have extremely reduced ability to regenerate spinal cord after injury. One of the exceptions are marsupials, such as opossums, that are born very immature, in an embryonic-like state (PO correspond roughly to E14-15 mouse or rat embryos), and they retain the ability to completely regenerate spinal cord after injury in the first two postnatal weeks $(54,55)$. It would be important to understand how the activation, proliferation and migration of EC after injury correlate with their neurogenic potential and postnatal spinal cord regeneration,possible in opossums and impossible in placental mammals. Recently, 
the transcription factor ATF3 was detected as a potential candidate to regulate SPC quiescence/activation in the spinal EC of rats as well as of the opossum Monodelphis domestica (27).

Together with currently available knowledge on placental mammals, comparative studies on regenerative species could provide important indications about the exact cell types responsible for the functional regeneration of the CNS tissue, and those results could contribute to provide a missing solution for the effective therapeutic strategy for CNS injuries and neuroregeneration.

\section{Conclusion}

Unlike in the brain, adult neurogenesis in the spinal cord is still under investigation, with unclear characterization of spinal SPC. Further efforts should be made to identify the "best" source of endogenous stem cells that could hopefully allow us to develop effective neuroregenerative strategies for SCI and neurodegenerative diseases.

\section{Acknowledgements}

The experimental work has been conducted on equipment financed by the European Regional Development Fund (ERDF) within the project "Research Infrastructure for Campus-based Laboratories at University of Rijeka" (RC.2.2.06-0001), the Croatian Science Foundation (CSF) grant IP-2016-06-7060 and the financial support from the University of Rijeka (18.12.2.1.01, 18-258-6427 and 18-290-1463).

\section{Conflict of interest}

The authors declare no conflict of interest.

\section{Funding body}

The experimental work has been conducted on equipment financed by the European Regional Development Fund (ERDF) within the project "Research Infrastructure for Campus-based Laboratories at University of Rijeka" (RC.2.2.06-0001), the Croatian Science Foundation (CSF) grant IP-2016-06-7060 and the financial support from the University of Rijeka (18.12.2.1.01, 18-258-6427 and 18-290-1463).

\section{References}

1. Badhiwala $\mathrm{JH}$, Ahuja CS, Fehlings MG. Time is spine: a review of translational advances in spinal cord injury. J Neurosurg Spine. 2018 20;30(1):1-18.

2. Courtine G, Sofroniew MV. Spinal cord repair: advances in biology and technology. Nat Med. 2019;25(6):898-908.

3. Trawczynski M, Liu G, David BT, Fessler RG. Restoring motor neurons in spinal cord injury with induced pluripotent stem cells. Front Cell Neurosci. 2019;13:369.

4. Ban J, Mladinić Pejatović M. Nanotechnology approaches for autologous stem cell manipulation in personalized regenerative medicine. In: Bodiroga-Vukobrat N, Rukavina D, Pavelić K, Sander GG, editors. Personalized Medicine in Healthcare Systems. Europeanization and Globalization, vol 5. Springer, Cham.

5. Casarosa S, Bozzi Y, Conti L. Neural stem cells: ready for therapeutic applications? Mol Cell Ther. 2014 Oct 15;2.

6. Stadtfeld M, Hochedlinger K. Induced pluripotency: history, mechanisms, and applications. Genes Dev. 2010 Oct 15;24(20):2239-63.

7. Hanna JH, Saha K, Jaenisch R. Somatic cell reprogramming and transitions between pluripotent states: facts, hypotheses, unresolved issues. Cell. 2010 Nov 12;143(4):508-25.
8. Takahashi K, Yamanaka S. Induction of pluripotent stem cells from mouse embryonic and adult fibroblast cultures by defined factors. Cell. 2006 Aug 25;126(4):663-76.

9. Takahashi K, Tanabe K, Ohnuki M, Narita M, Ichisaka T, Tomoda $\mathrm{K}$, et al. Induction of pluripotent stem cells from adult human fibroblasts by defined factors. Cell. $2007 \mathrm{Nov}$ 30;131(5):861-72.

10. Nagoshi N, Tsuji O, Nakamura M, Okano H. Cell therapy for spinal cord injury using induced pluripotent stem cells. Regen Ther. 2019 Dec;11:75-80.

11. Tsuji O, Sugai K, Yamaguchi R, Tashiro S, Nagoshi N, Kohyama J, et al. Concise Review: laying the groundwork for a first-in-human study of an induced pluripotent stem cell-based intervention for spinal cord injury. Stem Cells. 2019;37(1):6-13.

12. Yoshihara M, Hayashizaki Y, Murakawa Y. Genomic instability of iPSCs: challenges towards their clinical applications. Stem Cell Rev Rep. 2017 Feb;13(1):7-16.

13. Hou P, Li Y, Zhang X, Liu C, Guan J, Li H, et al. Pluripotent stem cells induced from mouse somatic cells by smallmolecule compounds. Science. 2013 Aug 9;341(6146):651-4.

14. Zheng J, Choi K-A, Kang PJ, Hyeon S, Kwon S, Moon J-H, et al. A combination of small molecules directly reprograms mouse fibroblasts into neural stem cells. Biochem Biophys Res Commun. 2016 15;476(1):42-8.

15. Biswas D, Jiang P. Chemically Induced Reprogramming of somatic cells to pluripotent stem cells and neural cells. Int $\mathrm{J}$ Mol Sci. 2016 Feb 6;17(2):226.

16. Qin $\mathrm{H}$, Zhao $\mathrm{A}$, Fu X. Small molecules for reprogramming and transdifferentiation. Cell Mol Life Sci. 2017 Oct 1;74(19):3553-75.

17. Gaudet AD, Fonken LK. Glial cells shape pathology and repair after spinal cord injury. Neurotherapeutics. 2018;15(3):554-77.

18. Orr MB, Gensel JC. Spinal cord injury scarring and inflammation: therapies targeting glial and inflammatory responses. Neurotherapeutics. 2018;15(3):541-53.

19. Liddelow SA, Barres BA. Reactive astrocytes: production, function, and therapeutic potential. Immunity. 2017 Jun 20; 46(6):957-67.

20. Ban J, Sámano C, Mladinic M, Munitic I. Glia in amyotrophic lateral sclerosis and spinal cord injury: common therapeutic targets. Croat Med J. 2019 Apr;60(2):109-20.

21. Hugnot JP, Franzen R. The spinal cord ependymal region: a stem cell niche in the caudal central nervous system. Front Biosci (Landmark Ed). 2011 Jan 1;16:1044-59.

22. Kriegstein A, Alvarez-Buylla A. The glial nature of embryonic and adult neural stem cells. Annu Rev Neurosci. 2009;32(1):149-84.

23. Schwartz JH, Siegelbaum SA, Hudspeth AJ. Principles of neural science, Fifth Edition. McGraw Hill Professional; 2013. $1761 \mathrm{p}$.

24. Morest DK, Silver J. Precursors of neurons, neuroglia, and ependymal cells in the CNS: What are they? Where are they from? How do they get where they are going? Glia. 2003;43(1):6-18.

25. Malatesta P, Appolloni I, Calzolari F. Radial glia and neural stem cells. Cell Tissue Res. 2008 Jan;331(1):165-78.

26. McDonough A, Martínez-Cerdeño V. Endogenous proliferation after spinal cord injury in animal models. Stem Cells Int. 2012;2012:387513.

27. Mladinic M, Bianchetti E, Dekanic A, Mazzone GL, Nistri A. ATF3 is a novel nuclear marker for migrating ependymal stem cells in the rat spinal cord. Stem Cell Res. 2014 May;12(3):815-27.

28. Sabourin J-C, Ackema KB, Ohayon D, Guichet P-O, Perrin FE,Garces A, et al. A mesenchymal-like ZEB1(+) nicheharbors dorsal radial glial fibrillary acidic proteinpositive stem cells in the spinal cord. Stem Cells. 2009 Nov;27(11):2722-33. 
29. Xia B, Yanai I. A periodic table of cell types. Development. 2019 Jun 27;146(12).

30. Paridaen JTML, Huttner WB. Neurogenesis during development of the vertebrate central nervous system. EMBO Rep. 2014 Apr;15(4):351-64.

31. Englund C, Fink A, Lau C, Pham D, Daza RAM, Bulfone A, et al. Pax6, Tbr2, and Tbr1 are expressed sequentially by radial glia, intermediate progenitor cells, and postmitotic neurons in developing neocortex. J Neurosci. 2005 Jan 5;25(1):247-51.

32. Rushing G, Ihrie RA. Neural stem cell heterogeneity through time and space in the ventricular-subventricular zone. Front Biol. 2016 Aug 1;11(4):261-84.

33. Martínez-Cerdeño V, Noctor SC. Neural progenitor cell terminology. Front Neuroanat. 2018 Dec 6;12.

34. Malatesta P, Götz M. Radial glia - from boring cables to stem cell stars. Development. 2013 Feb 1;140(3):483-6.

35. Merkle FT, Tramontin AD, García-Verdugo JM, AlvarezBuylla A. Radial glia give rise to adult neural stem cells in the subventricular zone. Proc Natl Acad Sci USA. 2004 Dec 14;101(50):17528-32.

36. Noctor SC, Flint AC, Weissman TA, Dammerman RS Kriegstein AR. Neurons derived from radial glial cells establish radial units in neocortex. Nature. $2001 \mathrm{Feb}$ 8;409(6821):714-20.

37. Verkhratsky A, Parpura V. Introduction to neuroglia Colloquium Series on Neuroglia in Biology and Medicine: From Physiology to Disease. 2014 Feb 24;1(1):1-74.

38. Gubert F, Zaverucha-do-Valle C, Pimentel-Coelho PM, Mendez-Otero R, Santiago MF. Radial glia-like cells persist in the adult rat brain. Brain Res. 2009 Mar 3;1258:43-52.

39. Levitt P, Rakic P. Immunoperoxidase localization of glial fibrillary acidic protein in radial glial cells and astrocytes of the developing rhesus monkey brain. J Comp Neurol. 1980 Oct 1;193(3):815-40

40. Marichal N, Reali C, Trujillo-Cenóz O, Russo RE. Spinal cord stem cells in their microenvironment: the ependyma as a stem cell niche. Adv Exp Med Biol. 2017;1041:55-79.

41. Verkhratsky A, Butt A. Introduction to glia. In: Glial Neurobiology. John Wiley \& Sons, Ltd; 2007. p. 1-12.

42. Spassky N, Merkle FT, Flames N, Tramontin AD, GarcíaVerdugo JM, Alvarez-Buylla A. Adult ependymal cells are postmitotic and are derived from radial glial cells during embryogenesis. J Neurosci. 2005 Jan 5;25(1):10-8.

43. Sevc J, Daxnerová Z, Haňová V, Koval' J. Novel observations on the origin of ependymal cells in the ventricular zone of the rat spinal cord. Acta Histochem. 2011 Feb;113(2):156-62.

44. Meletis K, Barnabé-Heider F, Carlén M, Evergren E, Tomilin N, Shupliakov O, et al. Spinal cord injury reveals multilineage differentiation of ependymal cells. PLoS Biol. 2008 Jul 22;6(7):e182.

45. Goodman T, Hajihosseini MK. Hypothalamic tanycytesmasters and servants of metabolic, neuroendocrine, and neurogenic functions. Front Neurosci. 2015;9:387.

46. Laywell ED, Rakic P, Kukekov VG, Holland EC, Steindler DA. Identification of a multipotent astrocytic stem cell in the immature and adult mouse brain. Proc Natl Acad Sci USA. 2000 Dec 5;97(25):13883-8.

47. Johansson CB, Momma S, Clarke DL, Risling M, Lendahl $U$, Frisén J. Identification of a neural stem cell in the adult mammalian central nervous system. Cell. 1999 Jan 8; 96(1):25-34.

48. Fiorelli R, Cebrian-Silla A, Garcia-Verdugo J-M, Raineteau $O$. The adult spinal cord harbors a population of GFAPpositive progenitors with limited self-renewal potential. Glia. 2013;61(12):2100-13.

49. Reynolds BA, Weiss S. Generation of neurons and astrocytes from isolated cells of the adult mammalian central nervous system. Science. 1992 Mar 27;255(5052):1707-10.

50. Pastrana E, Silva-Vargas V, Doetsch F. Eyes wide open: a critical review of sphere-formation as an assay for stem cells. Cell Stem Cell. 2011 May 6;8(5):486-98.

51. Weiss S, Dunne C, Hewson J, Wohl C, Wheatley M, Peterson AC, et al. Multipotent CNS stem cells are present in the adult mammalian spinal cord and ventricular neuroaxis. J Neurosci. 1996 Dec 1;16(23):7599-609.

52. Doetsch F, Caillé I, Lim DA, García-Verdugo JM, AlvarezBuylla A. Subventricular zone astrocytes are neural stem cells in the adult mammalian brain. Cell. 1999 Jun 11; 97(6):703-16.

53. Shihabuddin LS, Horner PJ, Ray J, Gage FH. Adult spinal cord stem cells generate neurons after transplantation in the adult dentate gyrus. J Neurosci. 2000 Dec 1;20(23):8727-35.

54. Mladinic M, Muller KJ, Nicholls JG. Central nervous system regeneration: from leech to opossum. J Physiol (Lond). 2009 Jun 15;587(Pt 12):2775-82.

55. Nicholls J, Saunders N. Regeneration of immature mammalian spinal cord after injury. Trends Neurosci. 1996 Jun;19(6):229-34. 